\title{
CFD SIMULATION OF FLOW PAST MAV WINGS
}

\author{
Pradeep Shetty $^{++}$, M.B.Subrahmanya ${ }^{+}$, D.S. Kulkarni ${ }^{+}$, B.N.Rajani ${ }^{+}$ \\ ${ }^{++}$Project Assistant, CTFD Division, CSIR-NAL, Bangalore \\ ${ }^{+}$Scientist, CTFD Division, CSIR-NAL, Bangalore
}

\begin{abstract}
Turbulent flow past low aspect ratio (AR) thin wing used for two different MAV (Micro Air Vehicles) configurations viz. Black Kite and Golden Hawk has been carried out in order to analyze their aerodynamic characteristics. The Reynolds (Re) number for these two wings based on the root chord are $2.4 \times 10^{5}$ and $1.72 \times 10^{5}$ respectively. These simulations have been carried out using the in-house flow solution code to solve the Unsteady Reynolds Averaged Navier Stokes (URANS) equations coupled to different turbulence models. The standard $k-\varepsilon$ model has been used to simulate the turbulence for the Black Kite wing. The influence of three different turbulence models (standard $k-\varepsilon$, SA and SST) in predicting the aerodynamic coefficient has been studied for the Golden Hawk configuration. In the present study the aerodynamic characteristics computed for the two wing configurations are compared with the CSIR-NAL experiments. The cross flow patterns and the tip vortex for the Golden Hawk wing are presented and discussed.
\end{abstract}

Keywords: Mulitiblock structured grid, URANS, Finite volume solver, Low Re flow, Turbulence model, Low aspect ratio wing, Black Kite, Golden Hawk

\section{NOMENCLATURE}

$\begin{array}{ll}C & \text { chord of the aerofoil } \\ R e & \text { flow Reynolds number based on } C \\ C_{p} & \text { non-dimensional pressure coefficient } \\ C_{l} & \text { lift coefficient } \\ C_{d} & \text { drag coefficient } \\ C_{m} & \text { moment coefficient about quarter chord } \\ \left\langle U_{i}\right\rangle & \text { phase-averaged velocity component } \\ \langle P\rangle & \text { phase-averaged pressure } \\ k & \text { turbulent kinetic energy }\end{array}$

\section{ABBREVIATIONS}

\begin{tabular}{|c|c|}
\hline$\varepsilon$ & dissipation of turbulent kinetic energy \\
\hline$\Delta v$ & cell volume \\
\hline$y+$ & non-dimensional wall normal distance \\
\hline$\mu$ & fluid viscosity \\
\hline$\mu_{t}$ & eddy viscosity \\
\hline$u_{i}$ & fluctuating velocity component \\
\hline$J$ & Jacobian of the transformation \\
\hline$B_{k}^{i} \beta_{k}^{i}$ & metric coefficients of transformation \\
\hline$S_{U}$ & momentum source terms in $i$-direc \\
\hline
\end{tabular}

$\begin{array}{llll}\text { CFD } & \text { Computational Fluid Dynamics } & \text { SA } & \text { Spalrat Allmaras } \\ \text { SIMPLE } & \text { Semi Implicit Method for Pressure-Linked Equation } & \text { SST } & \text { Shear Stress Transport } \\ \text { QUICK } & \text { Quadratic Upwind Interpolation for Convective Kinematics } & \text { RANS } & \text { Reynolds Averaged Navier Stokes }\end{array}$

\section{INTRODUCTION}

Micro Air Vehicles (MAVs) are unmanned autonomous miniature flying machines which man has tried to mimic from the biological fliers like insects and small birds. A single MAV or a swarm of MAVs can be used effectively for surveillance so as to measure or gather relevant reliable information in hostile environments. These MAVs require the ability to loiter for a long duration and also have an efficient maneuver capability both in open and confined space. In many situations, MAVs can provide reliable solutions which are also extremely cost-effective.

\footnotetext{
*Scientist, Computational \& Theoretical Fluid Dynamics Division
}

These MAVs usual have a maximal size of $30 \mathrm{~cm}$ and flight speed ranging between 12 to $14 \mathrm{~m} / \mathrm{s}$ with 30 minute endurance and weighing below $300 \mathrm{~g}$. Recently MAV's have gained interest and have been used for both military and civilian purpose. Two important challenging problems in design of MAV's are (i) low Reynolds number ( $\mathrm{Re}$ ) which results in unfavourable aerodynamic conditions to support controlled flight, and (ii) small physical dimensions, resulting in certain unfavourable scaling characteristics including structural strength, reduced stall speed, and low inertia. The small length and the low velocity resulting in a flight regime with a very low Reynolds number $\left(10^{4}<\operatorname{Re}<5 \times 10^{5}\right)$ pose challenges in aerodynamic design of MAVs. The aerodynamics of wings and wing sections in this low 
Reynolds number regime is one of the interesting and less understood aspects of MAVs. Several factors which contributed to this poor performance are lack of understanding of aerodynamics, structural mechanics and the propulsion system at the micro scale and also the inadequate knowledge on the visual guidance and navigation systems.

Aerodynamic design of MAVs, reported so far, have employed different kinds of efficient lift generation systems viz., fixed wing $[1,2]$, flapping wings [2, 3], flexible wing [4] and rotary wings [5] and or their combinations. The fixed-wing MAVs are commonly used because they are simple and easy to implement and they usually fly at the upper end of the low Reynolds number regime $\left(\operatorname{Re}>1 \times 10^{5}\right)[2$, $6]$. It is well known from literature [2, 7] that at low Reynolds number, the aerodynamic characteristics greatly depend on the wing geometry. In general the MAVs require aerofoil with small thickness and significant camber in order to have a better aerodynamic performance at low Reynolds number. Furthermore the small dimension of MAV demand wing with low aspect ratio. Literature [1, 2] shows that wing planforms which are rectangular, elliptical, circular or its variants are ideal for MAV application since they offer more lifting area.

\section{MATHEMATICAL MODELLING}

\subsection{Governing Equations}

The phase-averaged Navier Stokes equations for unsteady turbulent incompressible flow are written in non-orthogonal curvilinear coordinates with cartesian velocities as dependent variables in a compact form as follows:

Momentum transport for the cartesian velocity component $\left\langle U_{i}\right\rangle$ :

$$
\begin{aligned}
& \frac{\partial\left(\rho\left\langle U_{i}\right\rangle\right)}{\partial t}+\frac{1}{J} \frac{\partial}{\partial x_{j}}\left[\left(\rho\left\langle U_{i} U_{k}\right\rangle \beta_{k}^{j}\right)\right] \\
& +\frac{1}{J} \frac{\partial}{\partial x_{j}}\left[-\frac{\mu}{J}\left(\frac{\partial\left\langle U_{i}\right\rangle}{\partial x_{m}} B_{m}^{j}+\frac{\partial\left\langle U_{k}\right\rangle}{\partial x_{m}} \beta_{i}^{m} \beta_{k}^{j}\right)\right] \\
& +\frac{1}{J} \frac{\partial}{\partial x_{j}}\left[\langle P\rangle \beta_{i}^{j}-\rho\left\langle u_{i} u_{k}\right\rangle \beta_{k}^{j}\right]=S_{U_{i}}
\end{aligned}
$$

These momentum equations are further supplemented by the mass conservation or the so-called continuity equation.

\section{Mass conservation (Continuity):}

$$
\frac{\partial}{\partial x_{j}}\left(\rho\left\langle U_{k}\right\rangle \beta_{k}^{j}\right)=\mathbf{0}
$$

However, the equations 1 and 2 do not form a closed system due to the presence of the unknown turbulent Reynolds stress term $-\rho\left\langle u_{i} u_{j}\right\rangle$. The Reynolds stress tensor is computed using an appropriate eddyviscosity based turbulence model.

\subsection{Turbulence Modelling}

In eddy viscosity based turbulence models, the turbulent stress appearing in the Reynolds-Averaged equations is expressed in terms of the mean velocity gradients as following:

$$
-\rho\left\langle u_{i} u_{j}\right\rangle=\mu_{t}\left(\frac{\partial\left\langle U_{i}\right\rangle}{\partial x_{j}}+\frac{\partial\left\langle U_{j}\right\rangle}{\partial x_{i}}\right)-\frac{1}{3} \rho \delta_{i j}\left\langle u_{m} u_{m}\right\rangle
$$

where, $\delta_{i j}$ is the Kronecker delta and $m$ is the summing index over $m=1,2,3$. The term $\frac{2}{3} \rho k \delta_{i j}$ only ensures that the sum of the normal stresses is $2 k$ as per the definition of $k$, the turbulent kinetic energy $k=\frac{1}{2} \overline{u_{i} u_{j}}$. The eddy viscosity $\mu_{t}$ is assumed to be an isotropic scalar quantity whose value depends on the local state of turbulence. For the present work standard $k-\varepsilon$ model [8] is used to simulate the turbulence.

\subsection{Numerical Solution of Finite Volume Equation}

The present computation uses a multi-block pressure-based implicit finite volume algorithm RANS3D [9, 10] developed at the CTFD Division, CSIR-NAL Bangalore to solve the unsteady turbulent incompressible flow using a structured grid. An iterative decoupled approach similar to the SIMPLE algorithm [11], modified for collocated variable arrangement [12] is adopted to avoid the checkerboard oscillations of the flow variables. The system of linear equations derived from the finite volume procedure is solved sequentially for the velocity components, pressure correction and turbulence scalars using the strongly implicit procedure of Stone [13].

\section{RESULTS AND DISCUSSIONS}

\subsection{Computational Details}

Three dimensional flow simulations have been carried out for two different wing planforms viz. modified inverse Zimmerman (Black Kite) with a semi-span of $0.6 C$ and modified cropped delta wing 
(Golden Hawk) having 0.625 $C$ semi-span. The flow Reynolds number for the Black Kite wing has been fixed as $2.4 \times 10^{5}$ based on the root chord length $(C)$ and wind speed of $14 \mathrm{~m} / \mathrm{s}$. Whereas for the Golden Hawk wing the flow Reynolds number has been fixed as $2.2 \times 10^{5}$ based on the root chord and wind speed of $12 \mathrm{~m} / \mathrm{s}$. For the present analysis a H-H grid topology with two blocks (one for the suction and other for the pressure side) covered by $193 \times 63 \times 69$ grid points along the stream wise, normal and span wise directions respectively has been generated using the in-house grid generation code [14]. The wing tip is closed linearly to obtain zero thickness. The grid is stretched towards the wing surface and the near wall grid spacing is so adjusted that the value of $y+$ at the first near wall point is maintained to be around 35 . Typical view of the grid on the wing surface and the symmetry plane for the two planforms are shown in the Figure 1. The computational domain and the boundary conditions used for the present simulation are shown in Figure 2. The present 3D simulation uses the QUICK scheme [15] for convective flux discretization and a second order central difference scheme for pressure and viscous term coupled to standard $k$ - $\varepsilon$ turbulence model [8]. The eddy viscosity at far field is assumed to be ten times of the laminar viscosity.

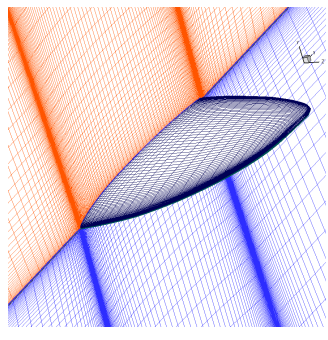

(a) Black Kite wing

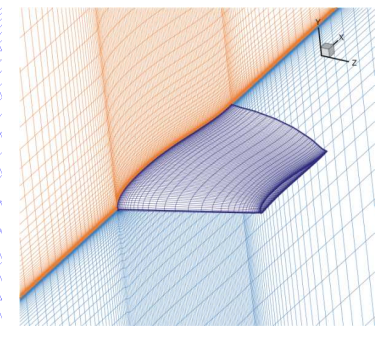

(b) Golden Hawk Wing
Figure 1. Grid on the symmetry plane and the wing surface

\subsection{Flow Past Black Kite Wing}

The flow analysis past the Black Kite wing has been carried out using the RANS3D code coupled to standard $k-\varepsilon$ turbulence model. The aerodynamic coefficients obtained from this simulation are compared with the NAL wind tunnel measurement data [16] for the full configuration (fuselage + wing + tail) as well as with the Fluent computation carried out by ADE, Bangalore [17] for the full configuration and are shown in Figure 3. The lift coefficient $\left(C_{l}\right)$ and drag coefficient $\left(C_{d}\right)$ computed along the flow axis from the RANS3D simulation (Figure 3(a) and 3(b)) is observed to agree reasonably well with the measurement data for the lower range of angles of

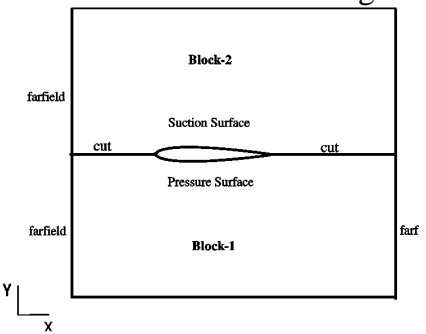

(a) X-Y plane

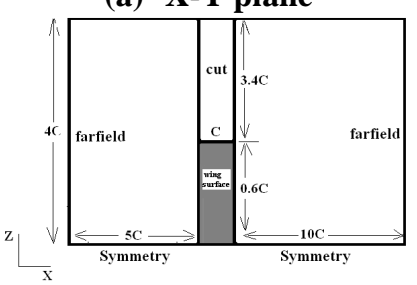

(b) X-Z plane

Figure 2. Boundary condition \& computational domain for $\mathrm{H}-\mathrm{H}$ grid topology

attack $\left(\alpha<15^{0}\right)$. Figure 3(c) clearly shows that the RANS3D has predicted a higher maximum lift-todrag ratio $\left((L / D)_{\max }=9.72\right)$ when compared to the experiments $\left((L / D)_{\max }=4.7\right)$ and the Fluent simulation $\left((L / D)_{\max }=6.12\right)$. The location of the maximum $L / D$ obtained by the RANS3D and Fluent simulations is almost same $\left(\alpha \approx 4^{0}\right)$ which slightly underpredicted when compared to the measurement $\left(\alpha \approx 7^{0}\right)$. The pitching moment $\left(C_{m}\right)$ computed along the body axis and about the leading edge of the wing from the RANS3D code (Figure 3 (d)) is observed to agree well with the measurement data at lower angles of attack. At higher angles of attack the present simulation has captured the trend but with a different slope when compared to the measurement. The difference in the $C_{m}$ values observed for the Fluent simulation may be the $C_{m}$ reported in the report [17] is calculated along the flow axis. The discrepancies observed at higher angles of attack may be due to the influence of the fuselage and the tail which has not been considered for the present simulation. In addition, on the computation side, this discrepancy may be due to the inadequate grid resolution and inability to model the transition. However the accuracy of the wind tunnel is not clearly quantified. Computations are also carried out for few typical angles of attack, at a flow velocity of $12 \mathrm{~m} / \mathrm{s}$ and no significant difference is observed in the aerodynamic coefficients for these two speeds. In order to sort out some of these issues flow analysis has been carried out for the Golden-Hawk wing using two different eddy viscosity based turbulence models viz. Shear 
Stress Transport (SST) model [18] and one equation Spalart-Allamaras (SA) model [19].

\subsection{Flow past Golden Hawk Wing}

The aerodynamic coefficients for the Golden Hawk wing obtained from the present simulation using three different turbulence models is compared with the NAL wind tunnel measurement data [16] for the full configuration (fuselage + wing + winglet) and is shown in Figure 4. The computed coefficient of lift (Figure 4(a)) agrees reasonably well with experimental data up to $\alpha=15^{\circ}$ and all the three turbulence models have predicted almost identical $C_{l}$ for $\alpha$ ranging between $-8^{0}$ to $18^{0}$. Beyond $18^{0}$ the SA and SST model have predicted higher $C_{l}$ when compared to the standard $k-\varepsilon$ model. Further the measurements have predicted a very early stall $(\alpha$ between $16^{\circ}$ and $17^{\circ}$ ) whereas the present computation has predicted the stall angle between $34^{\circ}$ and $36^{\circ}$. This early stall behaviour of the golden hawk wing is contradictor to the black kite wing where the measurement does not indicate the stall even up to $\alpha=25^{\circ}$. However the computation has predicted almost the same stall angle for both the configurations. Similar to the lift component the drag coefficient (Figure 4(b)) obtained from the RANS3D code matches well with the measurement data up to the stall angle and $C_{d}$ obtained by all the three models almost coincide with one another up to $\alpha=26^{\circ}$ beyond which the SA and SST have predicted a higher drag when compared to the standard $k-\varepsilon$ model. The lift-todrag ratio $(L / D)$ obtained by the present simulation using the standard $k-\mathcal{E}$ model and SST (Figure 4(c)) on the contrary matches fairly well with measurement data for the whole range of $\alpha$ with the maximum $L / D$ $(=7.6)$ over predicted as compared to measurement $(L / D=5.93)$. On the other hand, the SA model has grossly over predicted the maximum $L / D(=11.3)$ but the location at which the maximum $L / D$ occurs is same for all the three models $\left(\alpha=4^{0}\right)$ which slightly earlier than the measurement value $\left(\alpha \approx 6^{0}\right)$. However the coefficient of moment (Figure 4(d)) obtained from the present simulation using three different turbulence models is found to match well with the measurement data. The $C_{m}$ obtained experimentally shows a kink in the curve at the stall angle which is not seen in the computations. The discrepancies observed may be primarily attributed to the fact that the simulations have been carried out only for the wing configuration whereas the experiments have been carried out for the full configuration. On the computation side some more detailed analyses needs to be carried out by refining the grid size and using a different grid topology and also by modeling the effect of transition which play a prominent role at the low Reynolds number flows. In order to justify the accuracy of the present computation the comparison with the experiments for only the wing configuration would be more appropriate and due to lack of the measurement data this comparison exercise could not be carried out.

The non- dimensionalised pressure contours $\left(C_{p}=\left(p-p_{\infty}\right) / 1 / 2 \rho U_{\infty}^{2}\right)$ on the wing surface with the cross-flow patterns up to a length of $1.5 C$ in the wake are shown in the Figure 5 at three different angles of attack. The pressure distribution obtained is quite realistic with the reductions suction pressure as the angle of attack increases. The cross-flow pattern clearly indicates the complex vortex generated due to the flow past a finite wing which is observed to be more prominent at higher angles of attack. This vortex is observed to diffuse at the downstream stations. The diffusion of the vortex may be due to the coarse grid resolution in the wake.

Figure 6 shows the particle trace and the wing surface pressure contours typically at three angles of attack $\left(\alpha=4^{0}, 20^{\circ}\right.$ and $\left.30^{\circ}\right)$. The formation of the tip vortex is clearly evident especially at higher angles of attack. The wing tip vortices are caused due to the pressure difference between the upper surface (low pressure) and lower surface (high pressure) of the wing. This tip vortex induces a circulatory motion over the wing tip which affects the wing aerodynamics. In the downstream of the wing these tip vortices cause a secondary motion resulting in the forming of the wake vortex. The tip vortex is found to modify the pressure distribution of the wing [20] which in turn affects the lift-to-drag profile. In order to have a better estimate of the lift and drag coefficient it is necessary to capture the tip vortex and the intensity of this vortex greatly depends on how the wing tip is closed [20].

\section{CONCLUDING REMARKS}

The in-house RANS3D code has been successfully used to predict the aerodynamic loads and flow characteristics of MAV wings at relatively low Reynolds number. The aerodynamic loads obtained using the RANS3D code for the Black-Kite wing and Golden Hawk wing have been validated against NAL experiments. Reasonable agreement between the present computation and NAL experiments have been observed for both the configurations confirming the accuracy and adequacy of the flow solution algorithm. The discrepancies observed between the present computation and NAL 
experiments needs to be further investigated by refining the grid and changing the grid topology. Efforts are in progress to model the effects of the transition in the RANS framework which is expected

to give a better estimate of the aerodynamic characteristics especially for this low Reynolds number flows. The effect of closing the wing tip and its influence on the aerodynamic performance also needs to be investigated. The present simulation has successfully captured the wake flow and the tip vortex.

\section{ACKNOWLEDGMENTS}

The authors wish to thank Director CISRNAL and Head CTFD Division, Bangalore for permitting us to publish this paper. The authors also wish to express their heartfelt thanks to Head EAD Division, CSIR-NAL, Bangalore, D. Arivoli Scientist and MAV team of the EAD Division, CSIR-NAL, Bangalore for sharing the experimental data.

\section{REFERENCES}

1. J. M. Grameyer and M. T. Keennon. Development of the black Micro Air Vehicle. AIAA Paper, No. 2001-0127, 2001.

2. T. J. Mueller, "Fixed and flapping wing aerodynamics for micro air vehicles applications", Vol.195, Progress in Astronautics and Aeronautics, edited by T. J Mueller and published by AIAA, 2001.

3. G. W. Jones, C. J. Bradshaw, J. Papadapoulos, and M. F. Paltzer. Improved performance and control of flapping-wing propelled Micro Air Vehicles. AIAA Paper, No. 2004-0399, 2004.

4. W. Shyy, M. Berg, and D. Ljungqvist. Flapping and flexible wings for biological and micro vehicles. Progress in Aerospace Sciences, Vol. 35, No. 5, pp 455-506, 1999.

5. B. R. Hein. Hover performance of a Micro Air Vehicle: Rotors at low Reynolds number. AHS Lichten Award Competition, 2005.

6. M. S. Selig, J. J. Guglielmo, A. P. Broern, and P. Giguere. Experiments on Airfoils at Low Reynolds Numbers. 34th AIAA Aerospace Sciences Meeting and Exibit, Reno, NV, 1996.
7. R. T. Jones. "Wing Theory", Princeton University Press, 1990.

8. B. E. Launder and D. B. Spalding. The Numerical Computation of Turbulent Flows. Computer Methods in Applied Mechanics \& Engineering, Vol. 3, No. 2, pp. 269-289, 1974.

9. B. N. Rajani, A. Kandasamy and S. Majumdar. Numerical Simulation of Laminar Flow past a Circular Cylinder. Applied Mathematical Modelling, Vol. 33, pp.1228-1247, 2009.

10. S. Majumdar, B. N. Rajani, D. S. Kulkarni, and M. B. Subrahmanya. CFD simulation of low speed turbulent flow problems using unsteady RANS and Large Eddy Simulation approach. Journal of Aerospace Sciences and Technologies, 61:111-122, 2009

11. S. V. Patankar and D. B. Spalding. A calculation procedure for heat,mass and momentum transfer in three dimensional parabolic flows. International $\mathrm{Jl}$. of Heat and Mass Transfer, 15:1787-1806, 1972

12. S. Majumdar. Role of underrelaxation in momentum interpolation for calculation of flow with non-staggered grids. Numerical Heat Transfer, 13:125-132, 1988.

13. H. L. Stone. Iterative solution of implicit approximations of multidimensional partial differential equations. SIAM Jl. of Numerical Analysis, 5:530-530, 1968.

14. A. Fathima, N. S. Baldawa, S. Pal and S. Majumdar. Grid generation for arbitrary 2D configurations using a differential algebraic hybrid method. NAL PD CF9416, 1994.

15. B. P. Leonard. A stable and accurate convective modelling procedure based on quadratic interpolation. Computers Methods in Applied Mechanics and Engg., 19:59-98, 1979.

16. D. Arivoli and team, Pvt. Communication, EAD Division, CSIR-NAL, Bangalore

17. A. Vadivelan, S. Murugamohan, "CFD analysis of Black-Kite MAV", ADE report, Report No. : ADE/FSIM/MAV/Black-Kite-CFD-REP/001, $26^{\text {th }}$ April 2010. 
18. F. R. Menter. Two-equation eddy-viscosity turbulence models for engineering application. AIAA J, 32:269-289, 1994.

19. P. R. Spalart and S. R. Allamaras. A oneequation turbulence model for aerodynamic flow. AIAA paper, 92-0439, 1992.

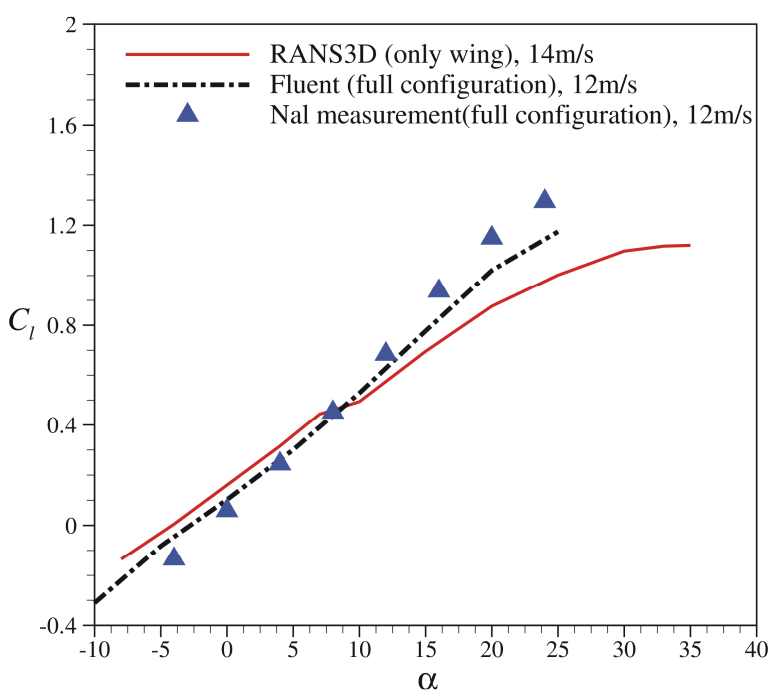

(a) Lift coefficient

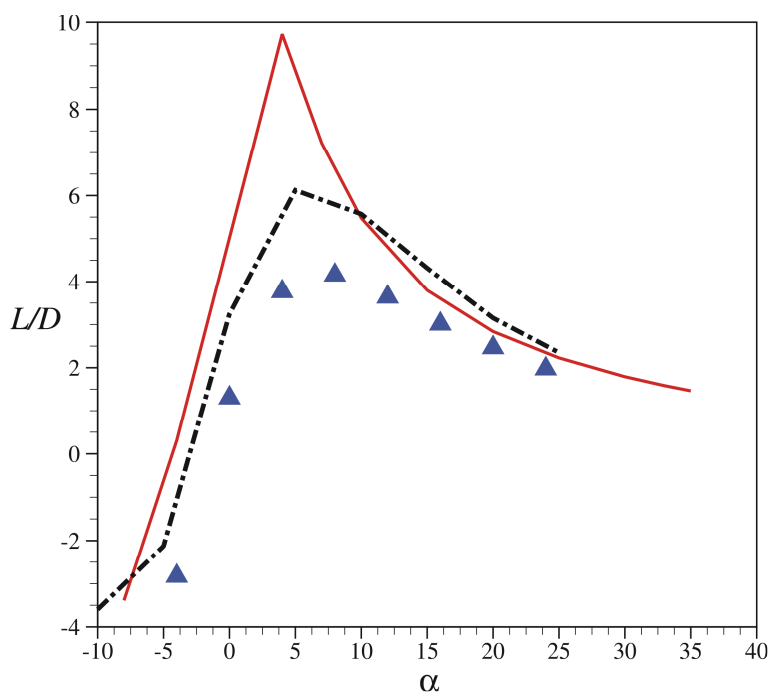

(c) Lift-to-drag ratio
20. D. Viieru, Y. Lien, W. Shyy and P. Ifju. Investigation of tip vortex on aerodynamic performance of a micro air vehicle. $33^{\text {rd }}$ AIAA Fluid Dynamic Conference and Exhibit, Orlando, Florida, 23-26 June 2003.

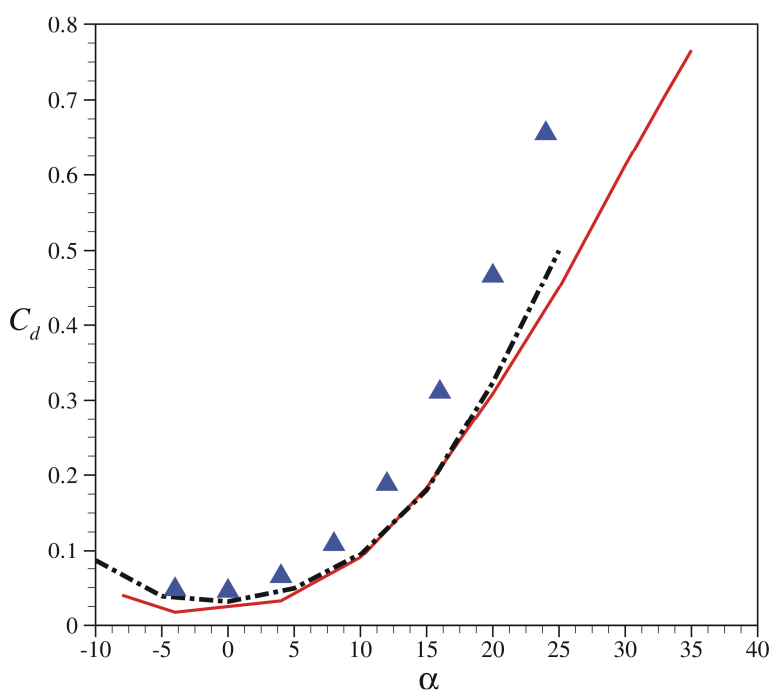

(b) Drag coefficient

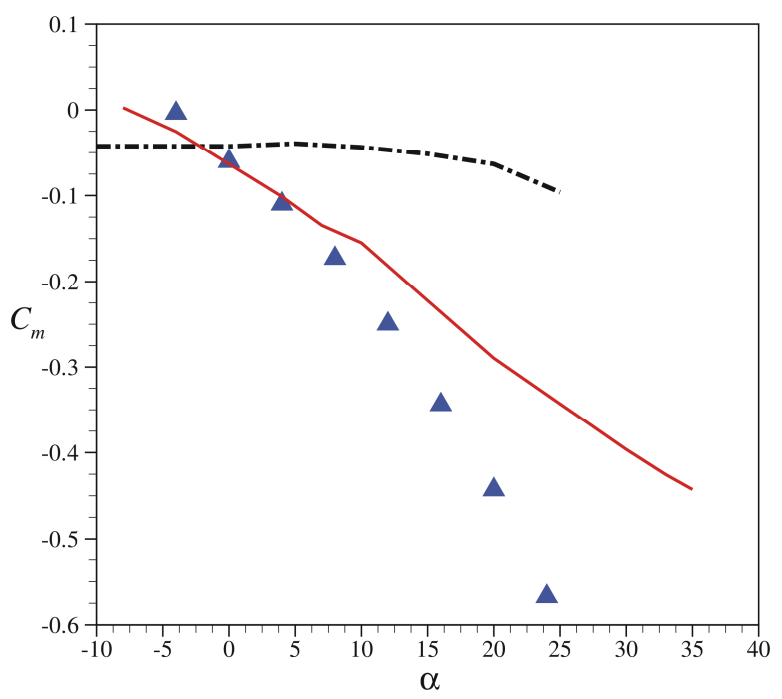

(d) Coefficient of moment

Figure 3. Aerodynamic coefficients for Black Kite wing $\left(\operatorname{Re}=2.4 \times 10^{5}\right)$ 


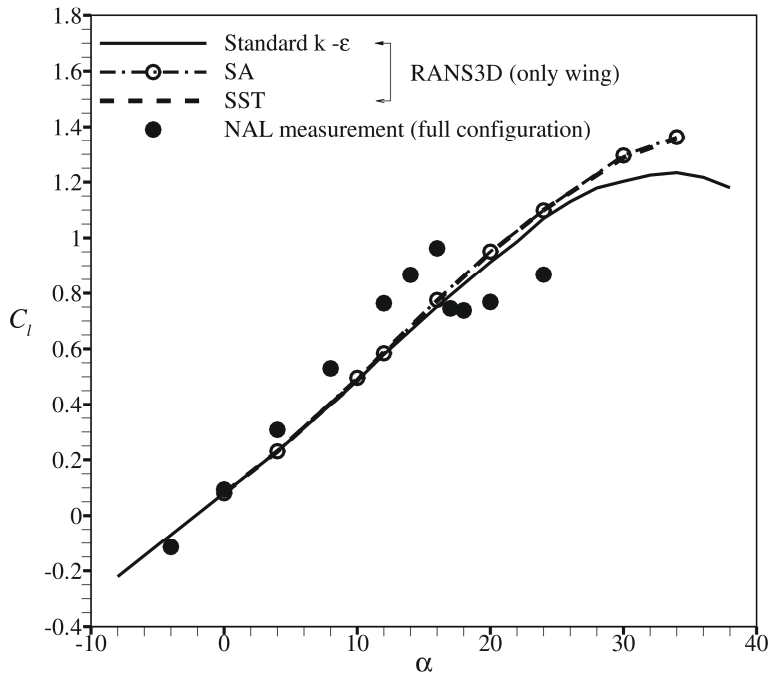

(a) Lift coefficient

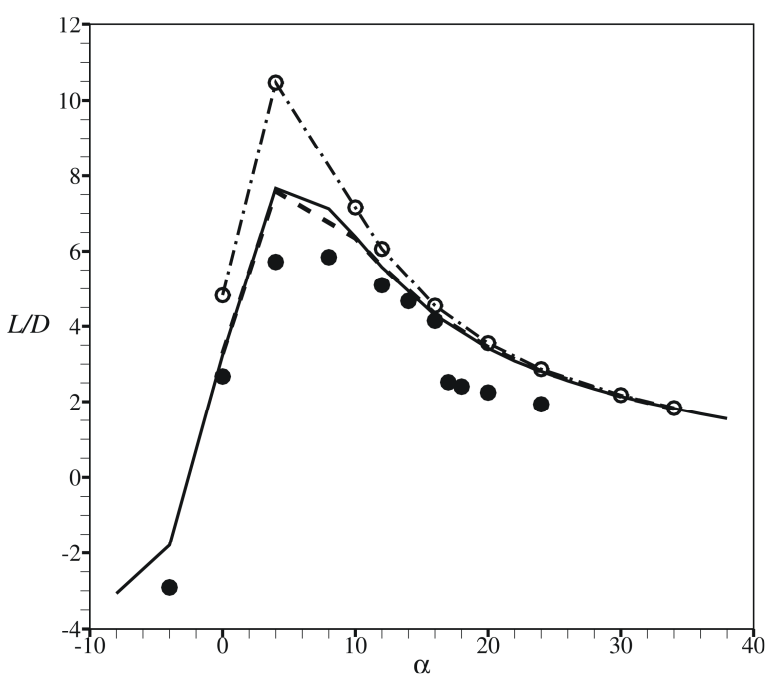

(c) Lift-to-drag ratio

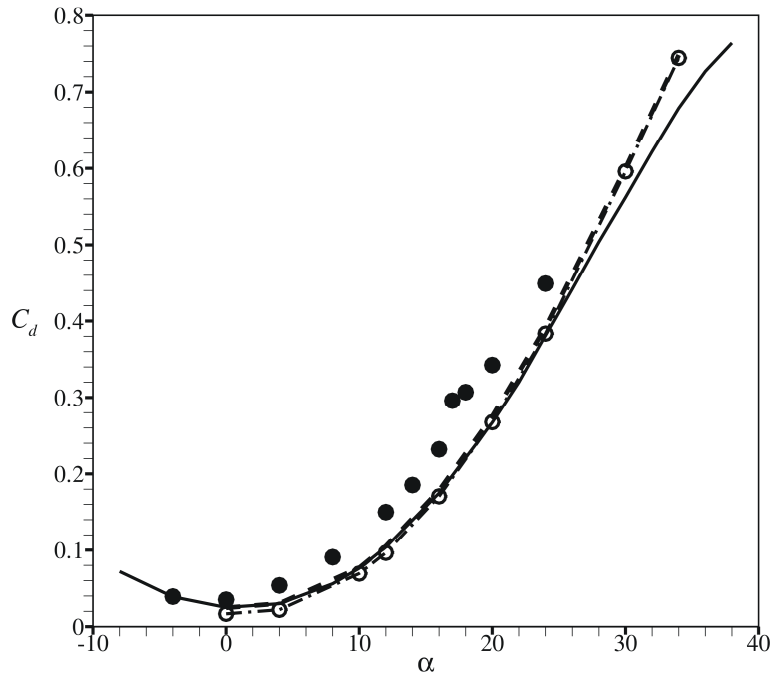

(b) Drag coefficient

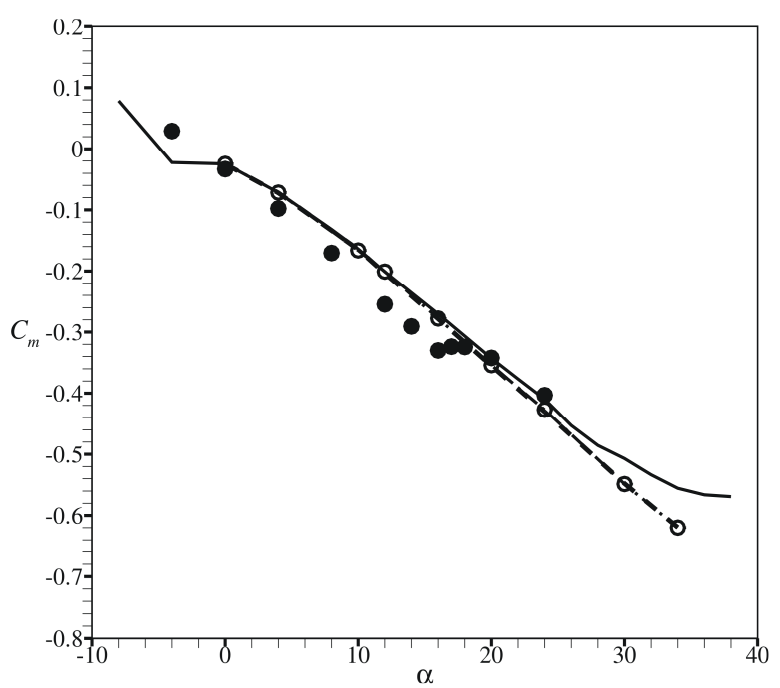

(d) Coefficient of moment

Figure 4. Aerodynamic coefficients for Golden Hawk wing $\left(\operatorname{Re}=1.7 \times 10^{5}\right)$ 


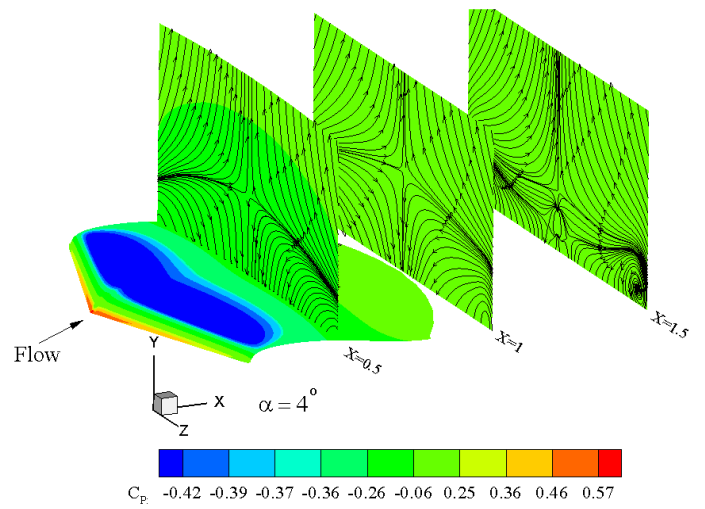

(a) $\alpha=4^{0}$

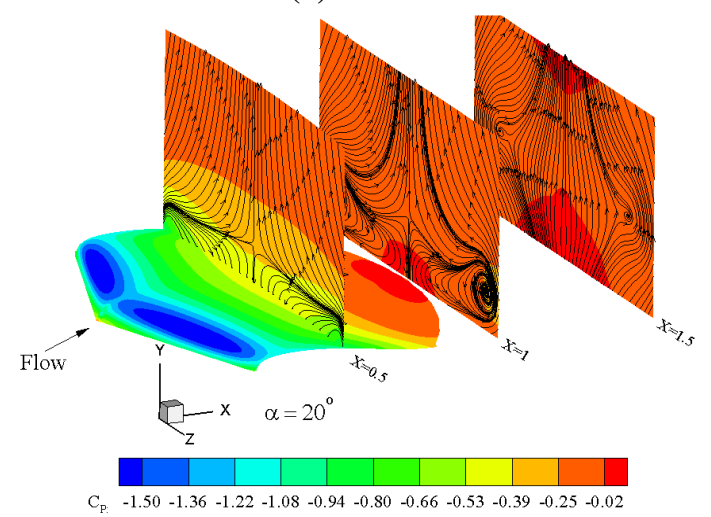

(b) $\alpha=20^{\circ}$

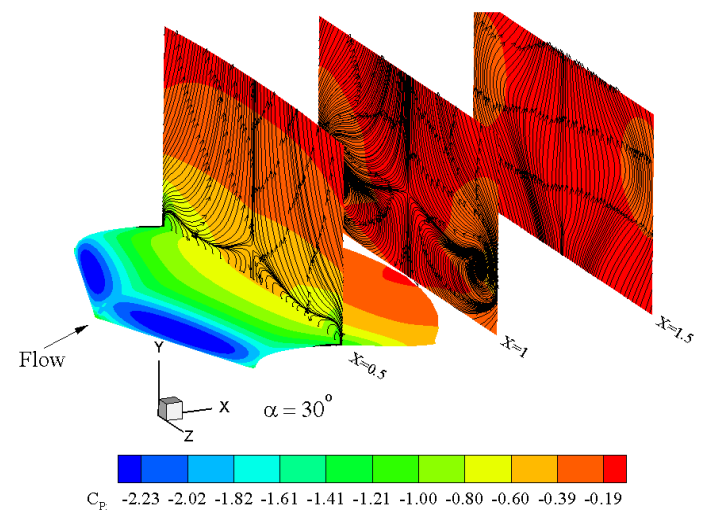

(c) $\alpha=30^{\circ}$

Figure 5. Surface pressure contour and crossflow pattern for Golden Hawk wing $\left(\operatorname{Re}=1.7 \times 10^{5}\right)$
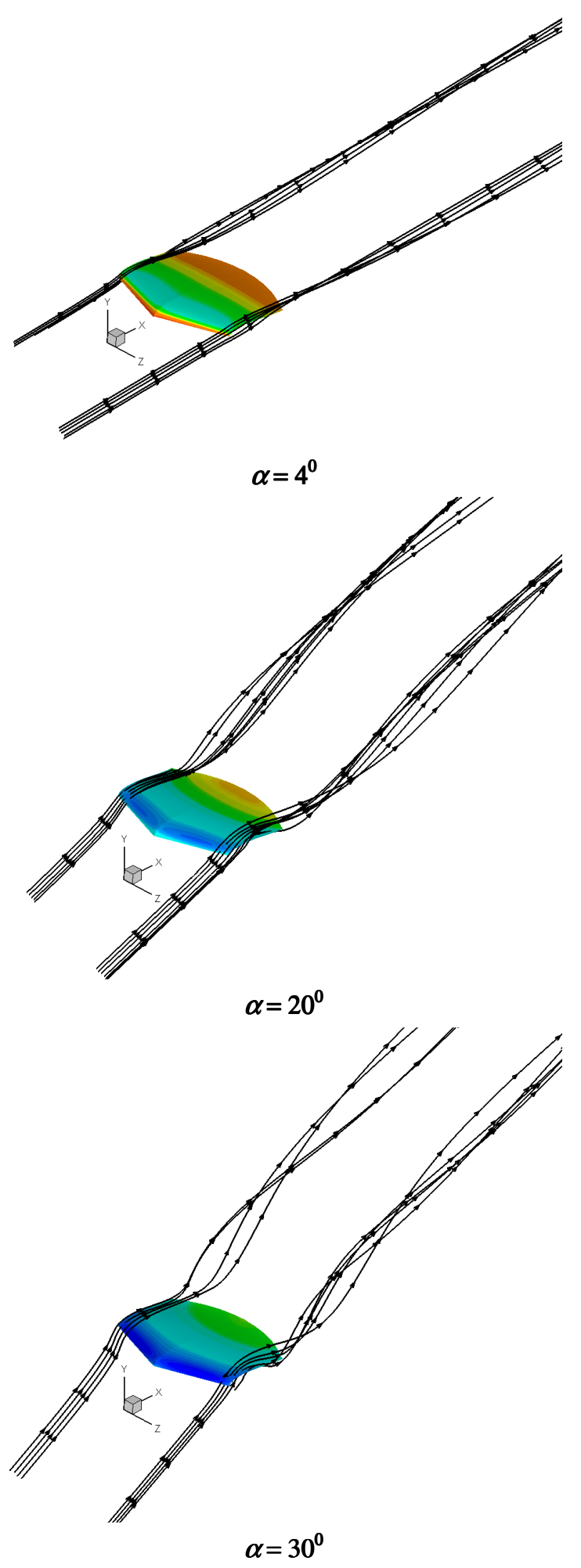

Figure 6. Particle trace for Golden Hawk wing showing the tip vortex $\left(\operatorname{Re}=1.7 \times 10^{5}\right)$ 